\title{
Effects of two types of feeding on the weight and open-field behavior of rats ${ }^{1}$
}

\section{DA VID LESTER, Wellesley College, Wellesley, Mass. 02181}

Rats were fed either in their cages or by placing food in receptacles outside but attached to the cage. The method of feeding had a significant effect on the ad lib weights of the rats but did not affect their open-field behavior.

The present study was designed to investigate the effects of throwing ad lib food into the cages of rats as opposed to placing the food in a wire receptacle attached to the outside of the cage, both of which methods are in common use. It was predicted that the former method would lead to larger weight gains in rats than the latter method. Such a difference would be of importance in studies where the deprivation of the animals was measured in terms of the percentage drop in weight from their ad lib weight. This technique of measurement would require refinement if the ad lib weight depended upon the method of feeding. Bolles (1967) discusses the merits of using percentage weight loss as an index of need.

The experiment was also designed to investigate whether the method of feeding had any effect on the open-field behavior of the rats. No prediction was made about the direction of such an effect.

\section{METHOD}

The Ss were 32 naive female rats from The Charles River Breeding Laboratories, Strain CD. They were 66 days old and housed singly in Wahmann cages $91 / 2$ in. $x 7$ in. $x 7$ in. They were given food and water ad lib.

The open field was a wooden table 27 in. $\times 42$ in. and elevated 31 in. It was divided into 21 rectangles each 6 in. $x$ 9 in.

The rats were weighed daily and received no other handling experience. On Day 5 the rats were divided into matched groups by weight. Group "In" continued to receive their food in their cage. Group "Out" received their food in a small feeding cup attached to the outside of the cage. These rats, therefore, had to struggle to obtain small pieces of food through the cage wire for eating. The rats were weighed daily through Day 33.

On Days 31 and 34 each of 20 rats (10 from Group In and 10 from Group Out) were placed for $5 \mathrm{~min}$ on the open field

Table 1

The Mean Activity (and Standard Deviations) of the Rats in the OpenField Test

\begin{tabular}{lll}
\hline & \multicolumn{1}{c}{ Day 1 } & \multicolumn{1}{c}{ Day 2 } \\
\hline Group OUT & $66.2(11.9)$ & $67.4(8.9)$ \\
Group IN & $72.8(11.9)$ & $66.8(22.8)$ \\
\hline
\end{tabular}

and the number of rectangles entered was noted. ${ }^{2}$ An entry was defined as made when the front two paws of the rat had entered a square. After each had been removed from the open field, the field was washed with water.

RESULTS

To compute the changes in weight, the mean weight of the rats on Days 28 and 29 was compared to the mean weight on Days 4 and 5 and expressed as a percentage increase over the weight on Days 4 and 5 .

The median percentage gain of the Out group was $28.3 \%$ (range $18.3 \%$ to $35.4 \%$ ) and the median percentage gain of the In group was $31.8 \%$ (range $23.2 \%$ to $52.9 \%$ ). This difference was significant (Mann-Whitney $U=76, z=1.97$, one-tailed $p=0.025$ ).

As a check on this difference, the percentage increase was calculated using the weight of the rats on Day 33. Again the difference was significant (Mann-Whitney $\mathrm{U}=78, \mathrm{z}=1.89$, one-tailed $\mathrm{p}=0.03$ ).

The mean weights of the two groups of rats did not differ on Days 4 and 5 (Mann-Whitney $U=116 \frac{1}{2}$, not significant).

One rat defecated in the open field (from the In group) and one rat fell off the table (from the Out group). The mean number of squares entered on each of the two trials (with the standard deviations) are shown in Table 1. The difference on Day 31 was not significant $(t=1.126, d f=18)$ nor on Day 34 $(t=0.08, d f=17)$. The differences were in opposite directions on the two days.

\section{DISCUSSION}

The differences in the weights of the rats obtained by the two methods of ad lib feeding was in the predicted direction, with those fed with food inside their cages gaining more. Thus, experiments concerned with the effects of specific deprivation percentages should take into account the particular method of ad lib feeding.

The failure to obtain an effect on open-field behavior from the method of feeding is perhaps postdictable. Montgomery (1953) found no effect from restriction in a small as opposed to a large cage on the exploratory behavior of rats. Therefore, there should be no effect on exploratory activity expected from the greater effort expended by the rats fed on the outside of the cage compared to those fed on the inside.

\section{REFERENCES}

BOLLES, R. C. Theory of motivation. New York: Harper \& Row, 1967 MONTGOMERY, K. C. The effect of activity deprivation upon exploratory behavior. Journal of Comparetive \& Physiological Psychology, 1953, 46, 438-441.

\section{NOTES}

1. This research was supported by Researeh Grant MH 14404-01 from the National Institute of Mental Health.

2. The 12 remaining rats had been assigned to a different experiment. 